\title{
Anorectal complications in patients with haematological malignancies
}

\author{
Rafaela V. Loureiro ${ }^{a}$, Verónica P. Borges ${ }^{a}$, Ana L. Tomé ${ }^{b}$, Carlos F. Bernardes ${ }^{a}$, Mário J. Silva and \\ Maria J. Bettencourt ${ }^{2}$
}

\begin{abstract}
Background Anorectal complications are common in patients with haematological malignancies.
Objectives The objectives are to characterize anorectal complications in these patients, identify risk factors and shed light on treatment, morbidity and mortality rates.
\end{abstract}
Patients and methods A retrospective, observational study that included 83 inpatients with haematological malignancies and proctological symptoms from January 2010 to September 2015 was conducted. Clinical outcomes were obtained through a detailed review of medical records.

Results The median age was 56 years, and 52 (62.7\%) patients were men. Fifty-six (67.5\%) patients had nonseptic anorectal complications and 27 (32.5\%) patients had septic anorectal complications.

Risks factors Patients with septic anorectal complications were more commonly male, older, and had lower absolute neutrophil counts, but the differences were not statistically significant $(P=0.79,0.67$ and 0.89 , respectively). In positive blood cultures [23/70 (32.9\%)], Enterococcus faecium, Klebsiella pneumonia, and Escherichia coli were the most common isolated agents.

Treatment In nonseptic anorectal complications, conservative treatments/minor proctological procedures were adopted, and patients with septic anorectal complications were treated with antibiotics \pm major proctological procedures and/or surgical drainage/debridement.

Results of treatment Forty-eight (85.7\%) patients in the nonseptic complications group improved compared with 23 (85.2\%) patients in the septic complications group. The overall mortality rate was $2.4 \%(n=2)$, with one $(1.2 \%)$ death related to perianal sepsis.

Conclusion Enterococcus spp. were more commonly identified in this study and can be increasing in this specific population. In contrast to other reports, we did not identify an association between septic anorectal complications and possible risk factors such as male sex, younger age or a low absolute neutrophil count. Most patients had nonseptic anorectal complications. A major proctological procedure/surgical debridement should always be applied in septic complications, which have better prognoses now than in the past. Eur J Gastroenterol Hepatol 00:000-000

Copyright @ 2018 Wolters Kluwer Health, Inc. All rights reserved.

\section{Introduction}

Anorectal complications in patients with haematological malignancies have long been known [1]. Palma [1] showed a relationship between haematological diseases and an increase in anorectal complications, with anorectal manifestations possibly being the first manifestations of haematological diseases.

Later, Schimpff et al. [2] studied a population of 581 patients and concluded that there is an increase in perianal/ perirectal infections in patients with haematological cancers compared with patients with nonhaematological cancers $(6.5$ vs. $0.5 \%)$.

\footnotetext{
European Journal of Gastroenterology \& Hepatology 2018, 00:000-000 Keywords: haematological malignancy, proctologic disease, risk factors Departments of ${ }^{a}$ Gastroenterology and ${ }^{b} H e m a t o l o g y$, Central Lisbon Hospital Center, Lisboa, Portugal

Correspondence to Rafaela V. Loureiro, MD, Santo António dos Capuchos Hospital - Central Lisbon Hospital Center, Alameda de Santo António dos Capuchos, 1169-050 Lisboa, Portugal

Tel/fax: +351 213136 320; e-mail: loureiro.rafaela@gmail.com
}

Received 21 October 2017 Accepted 10 March 2018
Moreover, the development of anorectal complications has been associated with worse prognosis, with a mortality rate of up to $50 \%$ [2-4].

Patients with haematological malignant diseases are immunocompromised and therefore particularly prone to anorectal complications. Multiple etiopathogenic factors have been identified: alterations in mucocutaneous barriers owing to haematological diseases or previous anorectal diseases, predisposition for bacterial invasion, some types of intestinal dysfunction such as diarrhoea or constipation (which can be related or unrelated to therapy), immunosuppression owing to loss of normal leucocyte function or chemotherapy, granulocytopenia with granulocyte counts lower than 500 cells $/ \mathrm{mm}^{3}$, thrombocytopenia that leads to delayed healing and opportunistic infections owing to immunosuppression [2,3,5-8].

The diagnosis of an anorectal complication in such patients may be challenging, especially when differentiating between septic or nonseptic complications. A careful proctologic examination should always be performed and should be complemented with radiological imaging (ultrasound or MRI) [8-10].

In the past, incision and drainage in such patients was controversial, owing to the fear of impaired healing in 
immunosuppressed patients, possibly aggravating the local disease progress. Supportive measures such as stool softeners, antidiarrhoeal agents, sitz baths, warm compresses, and antibiotics were the recommended treatments [2,3].

Several studies established the benefits of incision and surgical debridement as the preferable treatments in septic complications, in conjunction with supportive measures, and this is currently the recommended therapy $[5,8,10-12]$.

The evolution of local treatments associated with improvements in the supportive care of patients with neutropenic cancer resulted in a decrease in infectionrelated morbidity and mortality $[5,7,11,13]$.

The objectives of this study are to examine the treatment of a contemporary series of patients with malignant haematological diseases and anorectal complications, to identify the risk factors for anorectal complications in a contemporary series of these patients, to shed light on the results of treatment and to shed light on the actual mortality rate.

\section{Patients and methods}

\section{Patients}

We retrospectively reviewed the medical records of all patients admitted to the Haematology Department of Central Lisbon Hospital Center from January 2010 to September 2015 who had at least one proctological evaluation during the admission episode. This included only adult patients who were 18 years old or older.

All patients who were younger than 18 years or older than 80 years, had HIV 1 and 2 infections, had inflammatory bowel disease, were pregnant, did not have haematological malignancies, had other oncological diseases or showed incomplete data were excluded. Patients with proctological diseases diagnosed in the year before the admission were also excluded.

The study was approved by the ethics committee.

\section{Variables}

A detailed review of medical records was performed to retrieve demographic data and disease-related and treatment-related details regarding both haematological and proctological disorders.

Only blood cultures performed in the 3 days before and 3 days after proctological evaluations were considered. Blood cultures were performed for all patients with fevers or when their haematologist considered them necessary. The absolute neutrophil counts at the time of the development of the anorectal complications were recorded. A gastroenterologist expert in proctology performed the diagnoses of the anorectal complications based on clinical symptoms and physical examination. When necessary, the diagnosis was complemented by MRI, endoanal ultrasonography and/or examination under anaesthesia.

Anorectal complications were classified into two groups: nonseptic complications and septic complications. Nonseptic complications included anal fissures, haemorrhoidal diseases (including haemorrhoidal disease grades II, III and IV and haemorrhoidal trombosis) and anorectal ulcers. Septic complications included anorectal abscesses, perianal fistulas [including types 1 (intersphincteric fistula), 2 (trans-sphincteric fistula) and 3 (suprasphincteric fistula) of Park's classification] [14] and perianal cellulitis.
Inpatient and outpatient records were evaluated during a follow-up period of 30 days to assess the course and the resolution of anorectal complications.

\section{Statistical analysis}

Continuous variables were presented as medians and ranges. Categorical variables were presented as proportions.

Comparisons between nonseptic and septic complications according to age, sex, absolute neutrophil counts or positive blood cultures were performed using Fisher's exact test or $\chi^{2}$-test as deemed appropriate. A multivariate logistic regression analysis was performed and a significance criterion of $P$ less than or equal to 0.05 was defined for analysis.

All statistical analyses were performed using Microsoft Office Excel 2013 (Microsoft Corporation, Redmond, Washington, USA) and STATA 12.1 (StataCorp LP, College Station, Texas, USA).

\section{Results}

A total of 100 patients were observed at the proctology department during the allotted time period. Seventeen patients were excluded: one without a haematological malignancy, one over the age of 80 years, one with a HIV 1 infection, two with other concomitant oncological diseases, two with proctological diseases diagnosed in the year before the admission and 10 owing to incomplete data. None of the patients had inflammatory bowel disease or were pregnant. Thus, 83 inpatients with haematological malignant diseases and anorectal complications were included in the present analysis. Table 1 shows the patient baseline characteristics and haematological diseases.

Sixty-eight $(81.9 \%)$ patients had active haematological diseases, and the rest were in haematological remission and were admitted due to complications related to treatment. Eighty $(96.4 \%)$ patients underwent chemotherapy within the previous 15 days.

Twenty $(24.1 \%)$ patients had no neutropenia (absolute neutrophil count $\geq 1500$ cells), one $(1.2 \%)$ patient had mild neutropenia (absolute neutrophil count 1000-1500 cells), five $(6.0 \%)$ patients had moderate neutropenia

\begin{tabular}{lc}
\hline Table 1. Patient characteristics and haematological malignancy $(N=83)$ \\
\hline Characteristics & $n(\%)$ \\
\hline Age (years) & \\
Minimum & 20 \\
Maximum & 80 \\
Median & 56 \\
$<40$ & $22(26.5)$ \\
$\geq 40$ & $61(73.5)$ \\
Sex & \\
Male & $52(62.7)$ \\
Female & $31(37.3)$ \\
Haematologic disease & \\
Acute myeloid leukaemia & $54(65.1)$ \\
Chronic myeloid leukaemia & $1(1.2)$ \\
Acute lymphoblastic leukaemia/lymphoma & $12(14.5)$ \\
Common B cell & $4(4.8)$ \\
Pre-B cell & $3(3.6)$ \\
T cell & $5(6.0)$ \\
Non-Hodgkin's lymphoma Burkitt & $4(4.8)$ \\
Hodgkin's lymphoma & $1(1.2)$ \\
Non-Hodgkin's lymphoma & $11(13.3)$ \\
&
\end{tabular}


(absolute neutrophil count 500-1000 cells) and most patients $[57(68.7 \%)]$ had severe neutropenia (absolute neutrophil count $<500$ cells). Of the patients with severe neutropenia, 54 had fever $\left(T \geq 38.0^{\circ} \mathrm{C}\right)$.

Blood cultures were not considered for 14 of the patients because they were not performed in the 3 days before and 3 days after the proctological evaluation. Of the 70 patients for whom blood cultures were obtained at the time of anorectal symptoms, cultures were negative in $47(67.1 \%)$ patients and positive in $23(32.9 \%)$ patients. Positive blood culture organisms included: Enterococcus faecium in six $(26.1 \%)$ patients, Klebsiella pneumoniae in six $(26.1 \%)$ patients, Escherichia coli in five (21.7\%) patients, Enterobacter cloacae in two $(8.7 \%)$ patients and Acinetobacter baumannii, Pseudomonas aeruginosa, Stenotrophomonas maltophila and methicillin-resistant Staphylococcus epidermidis in one $(4.3 \%)$ patient each.

The main proctological complaints in patients with nonseptic anorectal complications were proctalgia [43 (76.8\%)], rectal bleeding [32 (57.1\%)] and prolapse [18 $(32.1 \%)]$; in the patients with septic anorectal complications, the main proctological complaints were proctalgia [26 $(96.3 \%)]$, perianal tumefaction $17(63.0 \%)]$ and discharge [12 (44.4\%)]. Diarrhoea was more common in patients with septic anorectal complications [seven $(25.9 \%)$ vs. $12(21.4 \%)]$ and constipation was more common in patients with nonseptic anorectal complications [12 $(21.4 \%)$ vs. one $(3.7 \%)]$.

Most patients [56 (67.5\%)] had nonseptic anorectal complications: anal fissures in $30(36.1 \%)$ patients; haemorrhoidal disease grades II, III or IV [including haemorrhoidal thrombosis, six $(7.2 \%)]$ in $22(26.5 \%)$ patients; and anorectal ulcers in four $(4.8 \%)$ patients. In this group, conservative treatments (stool softeners, antidiarrhoeal agents, sitz baths, warm compresses, oral or topic analgesics, venotropics or antibiotics) or minor proctological procedures (thrombectomy or polidocanol sclerotherapy) were adopted, with an improvement of symptoms or a complete resolution in most patients [48 $(85.7 \%)]$. At a 30 -day follow-up, seven $(12.5 \%)$ patients remained symptomatic and one $(1.8 \%)$ patient died of another cause (pneumonia).

Twenty-seven $(32.5 \%)$ patients had septic anorectal complications: perianal fistulas in $13(15.7 \%)$ patients (two suprasphincteric fistulas, five trans-sphincteric fistulas and six intersphincteric fistulas), anorectal abscesses in nine $(10.8 \%)$ patients and perianal cellulitis in five $(6.0 \%)$ patients. In this group, $12(44.4 \%)$ patients were treated with antibiotics and the remaining $15(55.6 \%)$ patients underwent fistula cannulation procedures and seton placements (and when indicated, fistulotomies) or surgical drainage/debridement in conjugation with antibiotherapy. At a 30-day follow-up, there was an improvement of symptoms or a complete resolution in $23(85.2 \%)$ patients. Three $(11.1 \%)$ patients remained symptomatic and one $(3.7 \%)$ died owing to perianal sepsis. The patient who died was a 41-year-old female with $P$. aeruginosa bacteremia, a very low neutrophil count ( 20 cells) and perianal cellulitis. Figure 1 shows the outline of therapeutic management and results.

The overall mortality rate was $2.4 \%$ (two deaths), with only one death $(1.2 \%)$ related to perianal sepsis.

Patients who had septic anorectal complications were more commonly male, were older and had lower absolute neutrophil counts, but no differences were statistically significant in the multivariate logistic regression: male sex [odds ratio $(\mathrm{OR})=1.152,95 \%$ confidence interval $(95 \%$ $\mathrm{CI})=0.414-3.208, P=0.786]$, younger age $(\mathrm{OR}=1.266$, $95 \% \mathrm{CI}=0.425-3.769, P=0.672)$, low absolute neutrophil count $(\mathrm{OR}=1.082,95 \% \quad \mathrm{CI}=0.360-3.250$, $P=0.889)$ and positive blood culture $(\mathrm{OR}=0.660,95 \%$ $\mathrm{CI}=0.227-1.919, P=0.446)$ (Table 2).

\section{Discussion}

Anorectal complications are more frequent in patients with haematological malignant diseases compared with patients with other cancers [1,2]. Early identification of predisposing factors, early diagnosis and prompt treatment are mandatory for reducing morbidity and mortality.

In our study, the major proctological complaints were very similar in patients with nonseptic and septic anorectal complications, with proctalgia being the most common complaint in both groups $(83.1 \%)$. The differential diagnosis based on patients' histories can be challenging and should be complemented with careful proctological examinations and radiological imaging [8-10].

All patients were examined by a gastroenterologist expert in proctology under mild sedation or, if necessary, under anaesthesia, and examinations were complemented with MRI or endoanal ultrasonography when necessary. None of the patients with nonseptic complications developed a septic complication during the follow-up period.

Most patients with anorectal complications had active haematological diseases or underwent chemotherapy within the previous 15 days, which seem to be risk factors for anorectal complications; however, these were not statistically analysed owing to a reduced number of patients in the 'haematologic disease in remission' and 'no chemotherapy' arms.

Previous reports suggest chemotherapy as a risk factor for anorectal complications [2,7].

E. faecium, K. pneumoniae and E. coli were the most common bacteria isolated from blood cultures $(73.9 \%)$, which was expectable owing to a combination of immunosuppression, colonisation of perianal skin by enteric flora and breaks in anorectal mucosal integrity $[6,8]$. The positivity for E. coli is similar to previous reports $[2,3,5,8,12,15]$; however, positive blood cultures for $P$. aeruginosa were more frequent in other previous studies $[2,5,8]$. This could be explained because most patients underwent antibiotherapy with a spectrum covering $P$. aeruginosa. In our series, Enterococcus spp. were more commonly identified than in previous reports $[2,3,5,8,12,15]$.

Others studies should be done to evaluate if Enterococcus spp. infections are increasing in this specific patient population.

Similar to other studies, most patients with haematological diseases and proctological complaints were diagnosed with nonseptic anorectal complications (67.5 vs. $32.5 \%$ ) such as anal fissures, haemorrhoidal diseases (including haemorrhoidal thrombosis) and anorectal ulcers $[3,4,10,15]$.

In the general population, haemorrhoidal diseases are more common than anal fissures [16,17]; however, in this series of patients with haematological malignancies, anal fissures were more frequent than haemorrhoidal diseases 


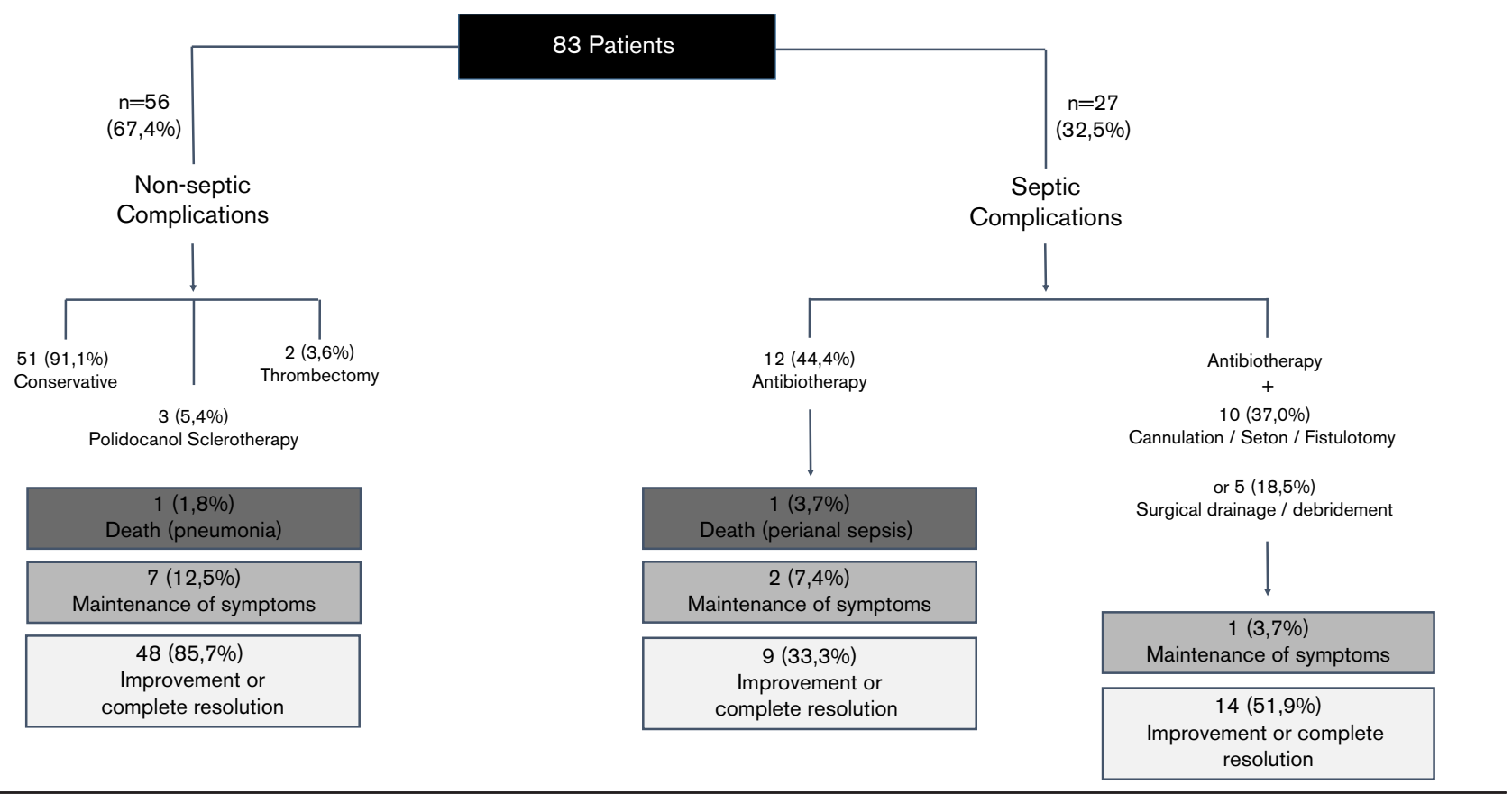

Fig. 1. Therapeutic management and results.

$\overline{\text { Table 2. Multivariate logistic regression analysis - risk factors for septic }}$ anorectal complications

\begin{tabular}{lccc}
\hline Risk factors & Odds ratio & $95 \%$ confidence interval & $P$ value \\
\hline Age $(<40$ years) & 1.266 & $0.425-3.769$ & 0.672 \\
Sex (male) & 1.152 & $0.414-3.208$ & 0.786 \\
ANC $(<500$ cells) & 1.082 & $0.360-3.250$ & 0.889 \\
Blood culture (positive) & 0.660 & $0.227-1.919$ & 0.446 \\
\hline
\end{tabular}

ANC, absolute neutrophil count.

(including haemorrhoidal thrombosis), 30 (36.1\%) vs. 22 $(26.5 \%)$, which is presumably owing to their haematological conditions and immunocompromised statuses.

In this group, conservative treatments or minor proctologic procedures were adopted, with either an improvement of symptoms or a complete resolution in most patients $(85.7 \%)$.

Twenty-seven $(32.5 \%)$ patients had septic anorectal complications such as perianal fistulas, anorectal abscesses and perianal cellulitis, and they were managed with antibiotherapy alone or antibiotherapy in conjunction with fistula cannulation procedures and seton placements (and when indicated, fistulotomies) or surgical drainage/debridement. Most patients $(85.2 \%)$ achieved either an improvement or a complete resolution of symptoms.

The only death associated with perianal sepsis occurred in a patient with perianal cellulitis in whom a surgical drainage/debridement could not be performed because of the severe neutropenia.

In our study, and similar to previous reports, early incision and surgical debridement in cases of patients with septic complications showed clear benefits and therefore should always be applied when appropriate $[5,8,10-12]$.

The overall mortality rate was $2.4 \%$, with only one $(1.2 \%)$ death related to perianal sepsis, which was very similar to recent studies and much lower than it was in the past. The improvement in outcomes in recent studies might be attributed not only to adequate management of anorectal complications but also to an improvement in the supportive care of patients with neutropenic cancer $[5,7,11,13]$.

In contrast to other studies, we did not identify an association between septic anorectal complications and possible independent risk factors such as male sex, a younger age, a low absolute neutrophil count or a positive blood culture. A relationship between septic anorectal complications and a younger age was only established in one study [10], but other studies showed a consistent association between a lower absolute neutrophil count and male sex and an increased incidence of septic anorectal complications in patients with haematological diseases $[2,4,8-10]$. The inability to demonstrate such associations may be owing to the small patient population.

All patients were examined in a single proctology department where the practices between different expert proctologists are very similar, which is a strong point of the study. However, this study has some potential limitations, as it is a single-centre study, has a retrospective study design and has a small study population.

As explained above, future studies aiming to identify the microorganisms implied in these conditions and other possible risk factors may have a significant effect in the identification of at-risk patients, thus improving management of these patients and preventing this condition.

This study demonstrates that nonseptic anorectal complications can be managed with conservative treatment; however, surgical debridement or proctologic procedures should often be applied in cases of septic complications, which have better prognoses now than in the past.

\section{Acknowledgements}

Authors' contributions: All authors contributed to the conception and design of study and to the acquisition, 
analysis and interpretation of data. All authors contributed to drafting the article or revising it critically for important intellectual content. All authors have approved the final version of the manuscript.

\section{Conflicts of interest}

There are no conflicts of interest.

\section{References}

1 Palma F. Afecciones anorrectales y hemopatias: aportación de três casos [Anorectal affections and hemopathies: contribution of three cases]. Rev Clin Esp 1963; 2:102-103.

2 Schimpff SC, Wiernik PH, Block JB. Rectal abscesses in cancer patients. Lancet 1972; 2:844-847.

3 Musa MB, Katakkar SB, Khaliq A. Anorectal and perianal complications of hematologic malignant neoplasms. Can J Surg 1975; 18:579-583.

4 Vanheuverzwyn R, Delannoy A, Michaux JL, Dive C. Anal lesions in hematologic diseases. Dis Colon Rectum 1980; 23:310-312.

5 Barnes SG, Sattler FR, Ballard JO. Perirectal infections in acute leukemia: improved survival after incision and debridement. Ann Intern Med 1984; 100:515-518.

6 Debat J, Prevost AG, Coliche V. Les lésions anorectales au cours des hémopathies. Ann GastroenterolHepatol 1986; 22:231-233.

7 Glenn J, Cotton D, Wesley R, Pizzo P. Anorectal infections in patients with malignant diseases. Rev Infect Dis 1988; 10:42-52.
8 Grewal H, Guillem JG, Quan SHQ, Enker WE, Cohen AM. Anorectal disease in neutropenic leukemic patients: operative vs nonoperative management. Dis Colon Rectum 1994; 37:1095-1099.

9 Badgwell BD, Chang GJ, Rodriguez-Bigas MA, Smith K, Lupo PJ, Frankowski RF, et al. Management and outcomes of anorectal infection in the cancer patient. Ann Surg Oncol 2009; 16:2752-2758.

10 Morcos B, Amarin R, Abu Sba' A, Al-Ramahi R, Abu Alrub Z, Salhab M. Contemporary management of perianal conditions in febrile neutropenic patients. Eur J Surg Oncol 2013; 39:404-407.

11 Corfitsen MT, Hansen CP, Christensen TH, Kaae HH. Anorectal abscesses in immunosuppressed patients. Eur J Sur 1992; 158:51-53.

12 Büyükasik Y, Ozcebe OI, Sayinalp N, Haznedaroglu IC, Altundag OO, Ozdemir $\mathrm{O}$, et al. Perianal infections in patients with leukemia: importance of the course of neutrophil count. Dis Colon Rectum 1998; 41:81-85

13 Lehrnbecher T, Marshall D, Gao C, Chanock SJ. A second look at anorectal infections in cancer patients in a large cancer institute: the success of early intervention with antibiotics and surgery. Infection 2002; 30:272-276.

14 Parks AG, Gordon PH, Hardcastle JD. A classification of fistula-in-ano. Br J Surg 1976; 63:1-12.

15 North JH, Weber TK, Rodriguez-Bigas MA, Meropol NJ, Petrelli NJ. The management of infectious and noninfectious anorectal complications in patients with leukemia. J Am Coll Surg 1996; 183:322-328.

16 Mapel DW, Schum M, Von Worley A. The epidemiology and treatment of anal fissures in a population-based cohort. BMC Gastroenterol 2014; $14: 1-7$.

17 Sanchez C, Chinn BT. Hemorrhoids. Clin Colon Rectal Surg 2011; 24:5-13. 